Journal of

\section{Synchrotron \\ Radiation}

ISSN 0909-0495

Received 25 April 2012

Accepted 13 August 2012

\title{
The point-spread function of fiber-coupled area detectors
}

\author{
James M. Holton, ${ }^{a, b *}$ Chris Nielsen ${ }^{c}$ and Kenneth A. Frankel ${ }^{b}$ \\ ${ }^{a}$ Department of Biochemistry and Biophysics, University of California, San Francisco, CA 94158- \\ 2330, USA, 'bawrence Berkeley National Laboratory, Berkeley, CA 94720, USA, and ${ }^{\mathbf{c}}$ Area \\ Detector Systems Corporation, Poway, CA 92064, USA. E-mail: jmholton@lbl.gov
}

\begin{abstract}
The point-spread function (PSF) of a fiber-optic taper-coupled CCD area detector was measured over five decades of intensity using a $20 \mu \mathrm{m}$ X-ray beam and $\sim 2000$-fold averaging. The 'tails' of the PSF clearly revealed that it is neither Gaussian nor Lorentzian, but instead resembles the solid angle subtended by a pixel at a point source of light held a small distance $(\sim 27 \mu \mathrm{m})$ above the pixel plane. This converges to an inverse cube law far from the beam impact point. Further analysis revealed that the tails are dominated by the fiber-optic taper, with negligible contribution from the phosphor, suggesting that the PSF of all fiber-coupled CCD-type detectors is best described as a Moffat function.
\end{abstract}

Keywords: protein crystallography X-ray detector; CCD; phosphor; fiber optic.

\section{Introduction}

The signal-to-noise ratio of weak diffraction spots is limited by the background-scattered photons that fall into the spotintegration area (Arndt \& Wonacott, 1977; Holton \& Frankel, 2010), and the size of this area is, in turn, limited by the pointspread function (PSF) of the detector. By definition, the PSF is the spatial distribution of the recorded signal when the detector is illuminated by an infinitesimally thin beam of light. In practice the PSF is usually measured with the smallest beam available and the shape of the incident beam is deconvoluted from the measured intensity distribution (see \$2.3).

It is often reported in the scientific literature and in detector product literature that the PSF of detectors is Gaussian (Madden et al., 2006), as would be expected from the central limit theorem (de Moivre, 1738; Hald, 1998), but if the PSF does not arise from a large number of independent random processes it may have a different shape. Some detectors have been found to produce Lorentzian-shaped peaks (Bourgeois et al., 1994), but the functional form of the PSF found here is different from both of these: an inverse cube law. One report (Schreurs et al., 2010) presented a functional form identical to that found here, but did not explain how it was obtained.

\subsection{Detector construction}

The detector studied here was an Area Detector Systems (Poway, CA, USA) model Quantum 315r (serial number 926). These detectors are a $3 \times 3$ tiled array of nine sub-detectors, each of which consists of a thermoelectrically cooled Amtel THX 7899 CCD chip hard epoxy bonded to the narrow end of a 3.7:1 glass fiber-optic taper (Incom, Charlton, MA, USA). The X-ray-sensitive phosphor is a thin layer $(\sim 20-40 \mu \mathrm{m})$ of powdered $\mathrm{Gd}_{2} \mathrm{O}_{2} \mathrm{~S}: \mathrm{Tb}(<5 \mu \mathrm{m}$ particles) sandwiched between the wide end of the taper and a thin aluminized black plastic front window. The CCDs and tapers are held in a vacuum, for which the front window is the seal. This vacuum seal holds the phosphor in place. X-ray photons pass though the front window and, upon absorption in the phosphor, $\sim 10 \%$ of the photon energy is converted into hundreds of visible-light photons, which are emitted in random directions. The visible photons scatter about among the phosphor particles (possibly reflecting off the inner surface of the front window) until ultimately being either absorbed or accepted into one of the optical fibers of the taper (reviewed by Gruner et al., 2002). Detailed simulations of this process have been conducted by Liaparinos et al. (2006).

The fiber-optic tapers consist of millions of thin $(10 \mu \mathrm{m}-$ diameter) optical fibers formed into a round billet that is then heated and pulled into an hourglass shape. This hourglass is then cut into two tapers, which are round, so packing them into a square array requires that the large end be cut down to a square. The narrow end of the taper will eventually be bonded to a CCD chip so must also be square, but care must be taken at this end not to over-cut and damage 'intact' fibers that actually directly connect to the phosphor end. A 'buffer zone' of about $5 \%$ of the total area is therefore left around the square 'intact region' on the narrow end. The fibers in this buffer zone are called 'severed' here because they terminate on one of the four lateral walls of the squared-off taper, nowhere near the phosphor. The intact region is made to be slightly smaller than the active area of the CCD to allow for small alignment errors when the CCD is bonded to the taper (see Fig. 1), so the CCD pixels around the edges of the chip are actually bonded to severed fibers. In this way, every point on 


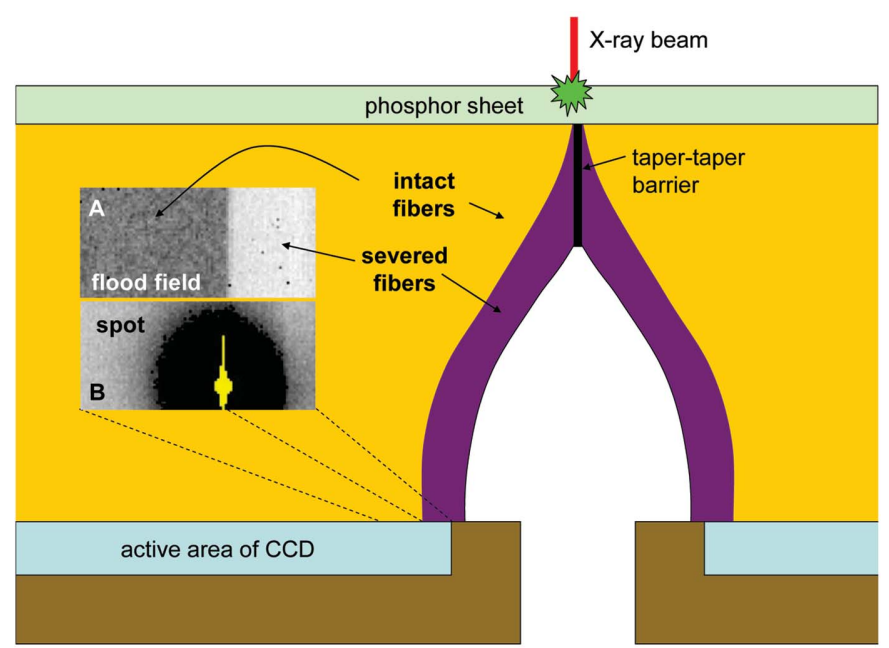

Figure 1

Schematic diagram of the detector construction. Yellow areas indicate 'intact' fibers which connect the CCD to the phosphor, and the purple region represents 'severed' fibers that contact the CCD but never reach the phosphor. Inset images are taken from the 'raw' CCD readout and demonstrate that light created by X-rays diffuses from the intact fibers into the severed fibers and onto the unused CCD pixels.

the phosphor face of the taper is directly connected to the $\mathrm{CCD}$, but not every point on the CCD is connected to phosphor. We took advantage of this quirk in the detector construction to separate the relative contribution of the fiberoptic taper and the phosphor to the total PSF.

\section{Methods}

\subsection{Experimental set-up}

A $50 \mu \mathrm{m}$-thick disk of tantalum (Ta) with a $15 \mu \mathrm{m}$ circular laser-drilled hole in the center was purchased from National Aperture (Salem, NH, USA), and placed at the sample position in the protein crystallography beamline 8.3.1 at the Advanced Light Source [instrument described by MacDowell et al. (2004)]. The photon energy was set to just above the Ta $L_{2}$-edge at $11141 \mathrm{eV}$ to maximize the stopping-power contrast of the pinhole, and the convergence angles of the beam were reduced to $50 \mu \mathrm{rad} \times 50 \mu \mathrm{rad}$ by adjusting a set of slits $10 \mathrm{~m}$ up-beam from the X-ray focus (pinhole position). The beam stop was removed and the Quantum 315r detector positioned $85 \mathrm{~mm}$ from the pinhole. After inserting absorbers, it was found that a $0.1 \mathrm{~s}$ exposure yielded a 'direct-beam' spot with peak pixel intensity of approximately 20000 pixel levels or 'analog to digital units' (ADUs) on an unbinned, dezingered and spatially corrected image. A total of 1883 such directbeam shots were collected, and each was followed by an equivalent 'explicit dark' exposure where the shutter was not opened. These explicit dark images were necessary because if the same dark image were subtracted from all the 'light' images then the noise in the common dark image would dominate the analysis below.

The distance between the pinhole and detector minimized the contributions of fluorescence and scattering from the pinhole to a negligible level (see \$3.2). Each image was collected with the detector driven to a slightly different position relative to the $\mathrm{X}$-ray beam: ranging at random over an area approximately one pixel wide and six pixels high (the pixel size was $51.3 \mu \mathrm{m}$ ). These movements were executed to sample more than just a single part of a single pixel on the detector surface, but at the same time involve only the central region of one fiber-optic taper. A $200 \times 200$ pixel region-ofinterest (ROI), centered on the spot, was extracted from each image and the corresponding explicit-dark image pixels subtracted to form a 'net' image with no read-out noise events in common with any other. The ROI was centered $2 \mathrm{~mm} \times$ $2 \mathrm{~mm}$ down and left of the center of the middle detector module, so all the pixels in this experiment were digitized by the same read-out channel.

Each net image was then fitted to a two-dimensional Gaussian function to roughly establish the fractional pixel coordinate of the center of the incident beam and also to obtain a rough scale factor from the height of each fitted function. Using these fitted parameters the midpoint of each pixel could then be assigned a linear distance from the 'beam center' and each spot put on a common scale with the others. These shifted and scaled data were then plotted as the red points in Fig. 2. Scale factors ranged from 0.75 to 1.35 and were due largely to variations in storage-ring current. Note that the grouping of pixel values at unity is a discretization artifact arising because the difference between any two integer-valued pixels must also be an integer.

To improve the signal-to-noise ratio in the low end, each pixel was treated as a square area of constant intensity and the intensity re-distributed onto a new common pixel grid using triangle binning with the program FIT2D (Hammersley, 1997). The resulting 'sum pixel' intensities are plotted as the blue

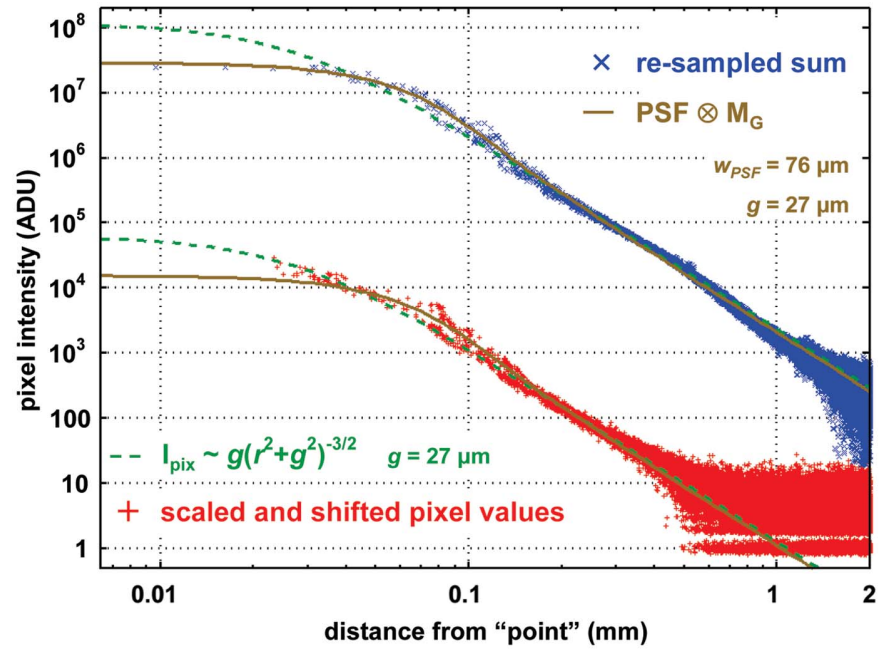

Figure 2

Scaled pixel intensities obtained by subtracting 'light' and 'dark' detector images are plotted as red points. The $x$-axis $(r)$ denotes the distance of the center of each pixel from the fitted center of intensity for the given pixel field. Blue points were obtained by re-binning and summing all intensity data, revealing that the inverse cube law continues out to at least $2 \mathrm{~mm}$ away from the beam impact point. The brown solid lines are the best fit to the PSF convoluted with a Gaussian and the green dashed lines are the 'deconvoluted' solid-angle component: function $P$. 
points in Fig. 2. This peak-fitting and re-binning procedure was repeated using the model for the actual PSF derived below to extract the center and scale of each observed spot, but the resulting changes to the points plotted in Fig. 2 were unremarkable.

\subsection{Assessment of fiber-optic taper contribution}

Separating the contributions of the phosphor and the fiberoptic taper to the PSF was achieved by moving the detector so that the $20 \mu \mathrm{m}$ X-ray beam hit the very edge of the center module and examining the 'raw' CCD readout image. This image contained the usual 'intact' pixels as well as the 'unused' pixels on the outer edges of the CCD chip (Fig. 1, inset B). The exposure was made long enough to just overload the central pixels so that the tails of the PSF would be significant. The intact CCD pixels (which are connected to the phosphor by intact fibers) were identified using a flood-field image (Fig. 1, inset A).

The pixel values from a trace through the image shown in Fig. 1 (inset A) are plotted in Fig. 3. It should be noted that the intact and severed curves in this figure are on the same scale, but the origin of the $x$-axis was arbitrarily chosen at 0.2 pixel widths from the center of the pixel with maximum intensity on the image. The actual fractional-pixel position of the beam impact point is obviously not available from the pixel values themselves, and extrapolating it by fitting a symmetric function to the nearby tails is not appropriate here because such a fit assumes that the PSF is identical in the intact and severed regions. However, this 0.2 pixel offset brings the two traces in Fig. 3 into close coincidence, demonstrating that the rate of fall-off in intensity is identical. A similar alignment could have been achieved by applying a scale factor to one of the curves (shifting all points up or down by the same distance on this log-scale graph), but this was not done. It is possible that there is a simple scale factor difference between the intact and severed regions, but it cannot be more than the intensity change produced by moving the beam by one pixel.

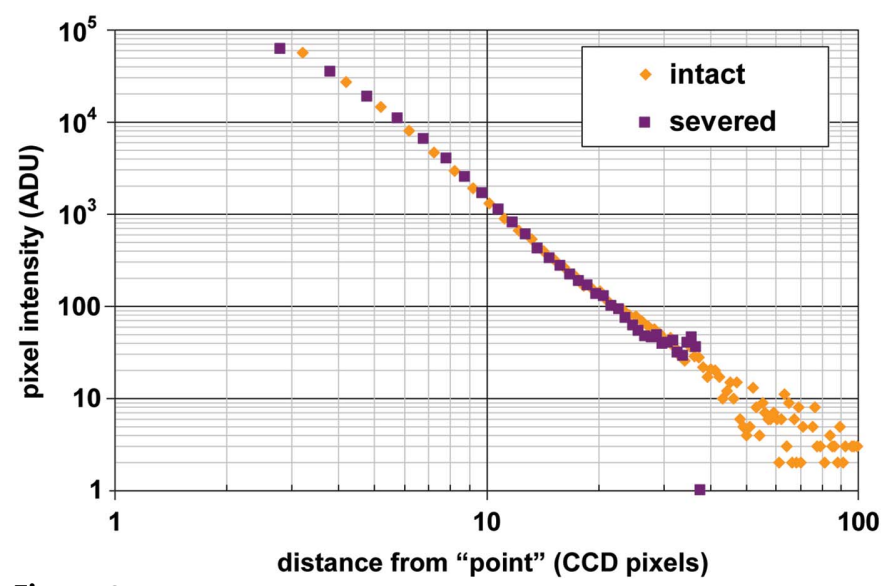

Figure 3

Comparison of relative intensity observed from CCD pixels attached directly to phosphor via intact fibers and those not connected to phosphor at all, except via scattering from the intact fibers. The similarity of intensity fall-off and overall scale indicates that the dominant component of the PSF tails is scattering between fibers in the taper.

\subsection{Mathematical representation of the PSF}

The pixel intensities observed here are not the 'true' PSF, but rather the convolution of the PSF with the beam profile, followed by integrating over the area of each pixel, so in this section we describe how these effects were decoupled. Specifically, the point spread observed here appears best described by a Moffat function (Moffat, 1969), which is essentially the convolution of a Gaussian with a power law. Unfortunately this convolution cannot be expressed in closed form, but the sum of a sufficient number of Gaussians can represent almost any function to within a desired error. A highly successful example of this approach is the popular '5-Gaussian' representation of atomic scattering factors (Vand et al., 1957; Cromer \& Waber, 1965; Maslen et al., 1999). The main utility of this representation is that convoluting atomic shapes with a Gaussian 'blur' may be performed analytically by simply adding the relevant $B$-factor to that of each of the component Gaussian terms.

For example, if both the PSF and the beam have Gaussian shapes, then the spot recorded on the detector will also be Gaussian, but with a full width at half-maximum (FWHM) related to that of the PSF ( $\left.w_{\mathrm{PSF}}\right)$ and beam $\left(w_{\text {beam }}\right)$ by

$$
w_{\mathrm{spot}}^{2}=w_{\mathrm{PSF}}^{2}+w_{\mathrm{beam}}^{2} .
$$

However, if either the beam or the PSF are not Gaussian, the convolution is not this simple. Suppose the PSF is still Gaussian but the X-ray beam profile is bimodal and, in effect, consists of two Gaussian 'sub-beams' with different $w_{\text {beam }}$ and intensity. In this case the spot recorded on the detector is the sum of the two spots one would observe with either sub-beam alone, using (1) to compute the FWHM of each 'sub-spot'. This treatment can be extended to an arbitrary number of subbeams, and theoretically any beam shape that can be 'painted' onto the detector face by using a variable Gaussian beam may be modeled with this formalism. In exactly the same way the spot profile resulting from a simple Gaussian beam and a nonGaussian PSF may be expressed as a sum of Gaussians if a suitable Gaussian-sum approximation to the PSF can be found.

Two-dimensional Gaussians may not have equal FWHMs in both directions, and indeed the major and minor axes may be tilted relative to the Cartesian coordinate system of the pixel plane. So, in general, two-dimensional Gaussians are convoluted by summing the elements of their covariance matrices.

The power-law component of the PSF found here appears to be of order 3, which resembles the solid angle subtended by a pixel at a point source of light some distance $g$ above the pixel plane,

$$
P(x, y)=\frac{g}{2 \pi\left(g^{2}+x^{2}+y^{2}\right)^{3 / 2}},
$$

where $x$ and $y$ are the Cartesian coordinates of a point of interest relative to the beam impact point. The integral of $P$ over the entire pixel plane is unity, reflecting how the energy of a single photon is divided amongst the pixels, and $P$ may be thought of as having units of 'intensity' per unit area. 
For comparison, the symmetric two-dimensional Gaussian with unit integral and unit FWHM is

$$
G(x, y)=\frac{\ln (16)}{\pi} \exp \left[-\ln (16)\left(x^{2}+y^{2}\right)\right] .
$$

We use $G$ to help represent the Gaussian component of the Moffat function,

$$
M_{G}(x, y)=\frac{1}{w_{\mathrm{PSF}}^{2}} G\left(\frac{x}{w_{\mathrm{PSF}}}, \frac{y}{w_{\mathrm{PSF}}}\right),
$$

which is still centrosymmetric and normalized to integrate to unity, but has FWHM $w_{\mathrm{PSF}}(\mu \mathrm{m})$. In turn, the X-ray beam may also be taken to have a Gaussian shape, but perhaps with different FWHM in the $x$ and $y$ directions ( $w_{\text {beam, } x}$ and $\left.w_{\text {beam, } y}\right)$,

$$
I_{\text {beam }}(x, y)=\frac{1}{w_{\text {beam }, x} w_{\text {beam }, y}} G\left(\frac{x}{w_{\text {beam }, x}}, \frac{y}{w_{\text {beam }, y}}\right) .
$$

Again the integral of $I_{\text {beam }}$ over the entire pixel plane is unity, since it represents the probability distribution of photon impact points.

Since both $M_{G}$ and $I_{\text {beam }}$ are Gaussians, their convolution $\left(M_{G} \otimes I_{\text {beam }}\right)$ may be computed analytically using (1) (see below), but the convolution $P \otimes M_{\mathrm{G}}$ cannot be expressed in closed form. We therefore approximate $P$ as the sum of a number of Gaussians,

$$
P_{n}(x, y)=\sum_{i=1}^{n} \frac{a_{i}}{g^{2} b_{i}^{2}} G\left(\frac{x}{g b_{i}}, \frac{y}{g b_{i}}\right),
$$

where $g$ is still the height of the 'point source' over the pixel plane, and $a_{i}, b_{i}$ are obtained by a fit of (6) to $P$ with the constraint that the sum of the volume of all $n$ Gaussian terms must be equal to 1 . An example of such coefficients with $n=8$ is given in Table 1 . Using these coefficients, $P_{n}$ matches $P$ to within $2.5 \%$ error over the six-decade range of the data available here.

Now, since addition and convolution commute, $P_{n} \otimes M_{G}$ may be expressed analytically as the sum of $n$ Gaussians, and used to approximate the full PSF $\left(P \otimes M_{G}\right)$. Furthermore, the full intensity spread $P \otimes M_{G} \otimes I_{\text {beam }}$ may also be approximated with only $n$ Gaussians. Specifically, we take each term in $P_{n}$ individually, and substitute the squared FWHM $\left(g^{2} b_{i}^{2}\right)$ with the sum of the squares of all the widths involved,

$$
\begin{aligned}
& w_{i, x}^{2}=g^{2} b_{i}^{2}+w_{\mathrm{PSF}}^{2}+w_{\text {beam }, x}^{2}, \\
& w_{i, y}^{2}=g^{2} b_{i}^{2}+w_{\mathrm{PSF}}^{2}+w_{\text {beam }, y}^{2},
\end{aligned}
$$

where $w_{\mathrm{PSF}}$ is the FWHM of the Gaussian component of the PSF, $w_{\text {beam }, x}$ and $w_{\text {beam, } y}$ are the FWHM of the beam in the $x$ and $y$ directions, and $w_{i, x}$ is the FWHM of the $i$ th Gaussian term in the approximation of $I_{\text {point }}$, the intensity per unit area deposited by a photon at any point in the pixel plane,

$$
\begin{aligned}
I_{\text {point }}(x, y) & =P \otimes M_{G} \otimes I_{\text {beam }} \\
& \simeq \sum_{i=1}^{n} \frac{a_{i}}{w_{i, x} w_{i, y}} G\left(\frac{x}{w_{i, x}}, \frac{y}{w_{i, y}}\right) .
\end{aligned}
$$

Table 1

Coefficients used to approximate $P$ with $P_{n}$ when $n=8$.

\begin{tabular}{lllllllll}
\hline & $i$ & \multicolumn{8}{l}{} & \multicolumn{1}{l}{} \\
\cline { 2 - 9 } & 1 & 2 & 3 & 4 & 5 & 6 & 7 & 8 \\
\hline$a_{i}$ & 0.1245 & 0.3170 & 0.2540 & 0.1474 & 0.07857 & 0.04120 & 0.02243 & 0.01494 \\
$b_{i}$ & 1.1401 & 2.1722 & 4.182 & 8.070 & 15.57 & 30.08 & 59.09 & 135.96 \\
\hline
\end{tabular}

If the beam shape is more complicated than a simple Gaussian, then it too may be represented as the sum of a collection of weighted Gaussian functions, much in the same way $P$ is approximated by $P_{n}$. That is, any beam profile may be represented as the sum of a collection of $m$ Gaussian-shaped subbeams. Replacing each of these sub-beams with (8) yields a total of $\mathrm{nm}$ Gaussian terms, representing the observed spot shape on the detector. In this work, however, we restricted our representation to a simple Gaussian-shaped beam.

Now, since we find below that $g=27 \mu \mathrm{m}, w_{\mathrm{PSF}}=76 \mu \mathrm{m}$ and the pixel size $\left(\ell_{\text {pix }}\right)$ is $51.27 \mu \mathrm{m}$, we expect that the value of $I_{\text {point }}$ will vary significantly from one side of a pixel to the other. Simply evaluating $I_{\text {point }}$ at the center coordinate of the pixel will not be a faithful representation of the real detector behavior, which is to integrate $I_{\text {point }}$ over the whole area of the spot. Algorithmically, this may be combated by dividing each pixel into many sub-pixels and averaging, but a much more elegant and computationally expedient approach is to take advantage of the analytic expression for the integral of a Gaussian over a square,

$$
H=\iint G \mathrm{~d} x \mathrm{~d} y=\frac{1}{4} \operatorname{erf}\left\{\frac{x[\ln (16)]^{1 / 2}}{w_{x}}\right\} \operatorname{erf}\left\{\frac{y[\ln (16)]^{1 / 2}}{w_{y}}\right\}
$$

where erf is the Gauss error function, and $w_{x}, w_{y}$ are still the FWHM of ' $G$ ', the Gaussian peak being integrated. The signal expected from a pixel is computed by taking the differences between the values of $H$ at the pixel corners,

$$
\begin{aligned}
G_{\mathrm{pix}}(x, y)= & H\left(x+\ell_{\mathrm{pix}} / 2, y+\ell_{\mathrm{pix}} / 2\right) \\
& +H\left(x-\ell_{\mathrm{pix}} / 2, y-\ell_{\mathrm{pix}} / 2\right) \\
& -H\left(x+\ell_{\mathrm{pix}} / 2, y-\ell_{\mathrm{pix}} / 2\right) \\
& -H\left(x-\ell_{\mathrm{pix}} / 2, y+\ell_{\mathrm{pix}} / 2\right),
\end{aligned}
$$

where $\ell_{\text {pix }}$ is the linear dimension of the edge of the square pixel $(\mu \mathrm{m})$ and $x$ and $y$ are the Cartesian coordinates of the pixel center relative to the beam impact point. Note that as $\ell_{\text {pix }}$ becomes large relative to $w_{x}$ and $w_{y}$ the value of $G_{\mathrm{pix}}(0,0)$ approaches unity, but as $\ell_{\text {pix }}$ becomes small relative to $w_{x}$ and $w_{y}$ the value $G_{\text {pix }}(x, y)$ approaches $G(x, y) \ell_{\text {pix }}^{2}$. We may now represent the fraction of the incident beam energy deposited into a given pixel $\left(I_{\text {pix }}\right)$ by the convolution of a Gaussian beam shape with our Moffat function PSF integrated over a pixel centered at $x, y$ relative to the beam center,

$$
I_{\mathrm{pix}}(x, y)=\sum_{i=1}^{n} \frac{a_{i}}{w_{i, x} w_{i, y}} G_{\mathrm{pix}}\left(\frac{x}{w_{i, x}}, \frac{y}{w_{i, y}}\right)
$$


where $a_{i}$ are taken from Table 1 and $w_{i, x}$ are taken from $b_{i}$ in Table 1 via (7). This function is represented by the brown lines in Fig. 2.

\section{Results and discussion}

\subsection{X-ray beam effects}

The beam shape at the detector surface was expected to be $\mathrm{a} \sim 20 \mu \mathrm{m}$-diameter rounded top-hat profile. The uncollimated beam profile at the pinhole position is a Gaussian of $\sim 120 \mu \mathrm{m}$ $\times 120 \mu \mathrm{m}$ FWHM (not shown), and so is essentially flat across the $15 \mu \mathrm{m}$-diameter Ta pinhole. The intensity distribution passing through the pinhole must therefore be a $15 \mu \mathrm{m}$ top-hat disk, dropping from $100 \%$ transmission to $\sim 1$ part per billion (through $50 \mu \mathrm{m} \mathrm{Ta}$ ) across the wall of the hole. This shape must be convoluted with the $4.2 \mu \mathrm{m}$ square top-hat distribution arising from the $50 \mu$ rad-wide distribution of beam directions (divergence) at the detector distance of $85 \mathrm{~mm}$. In addition, any irregularities in the walls of the hole must also be convoluted with the beam shape, but these are expected to be much smaller than $4 \mu \mathrm{m}$, so we assume here that the divergence-induced broadening dominates, giving a beam width at the baseline of $\sim 20 \mu \mathrm{m}$. This beam shape is less than half of the diameter of a pixel, but it should be noted that the PSF measured here is the convolution of the true PSF and this $\sim 20 \mu \mathrm{m}$-wide smoothed top-hat function. This deconvolution was achieved by the Gaussian decomposition described above.

The maximum possible fluorescence from the pinhole is dictated by the number of incident photons absorbed in the pinhole substrate (the Ta metal in which the hole was drilled). With a $120 \mu \mathrm{m}$ FWHM Gaussian incident beam, 368 photons are absorbed in the pinhole substrate for every photon passing through a $15 \mu \mathrm{m}$-diameter hole (ratio of the numerical integral of the two-dimensional Gaussian, in and out of the central hole). If all of these $11141 \mathrm{eV}$ photons are absorbed, then $30 \%$ and $60 \%$ will be taken up by the $L_{2}$ and $L_{3}$-edges, respectively, and $\sim 25 \%$ of each of these absorptions will result in a fluorescent X-ray photon emission (McMaster et al., 19691970). Since the solid angle subtended by a $50 \mu \mathrm{m}$ detector pixel at $85 \mathrm{~mm}$ from the pinhole position is $3.5 \times 10^{-7}$ sterad, we expect one fluorescent background photon per pixel for every $4.3 \times 10^{5}$ 'spot' photons. This is assuming no attenuation of the fluorescent photons $(8-9 \mathrm{keV})$, which would actually be stopped $4.7 \times 10^{5}$-fold by $50 \mu \mathrm{m}$ of Ta. No doubt a fraction of fluorescence generated in the walls of the hole does pass through the hole, but these photons originate from a very small fraction of the total incident photons, and thus the contribution of fluorescent photons to the net signal in the ROI is negligible over our six-decade intensity range of interest.

Bragg scattering from the pinhole will obviously be negligible as the first diffraction order from crystalline Ta is well outside the $200 \times 200$ pixel region of interest used here. Smallangle X-ray scattering (SAXS) from the pinhole walls may have some contribution, but this is difficult to predict without knowing the microscopic grain structure of the laser-drilled

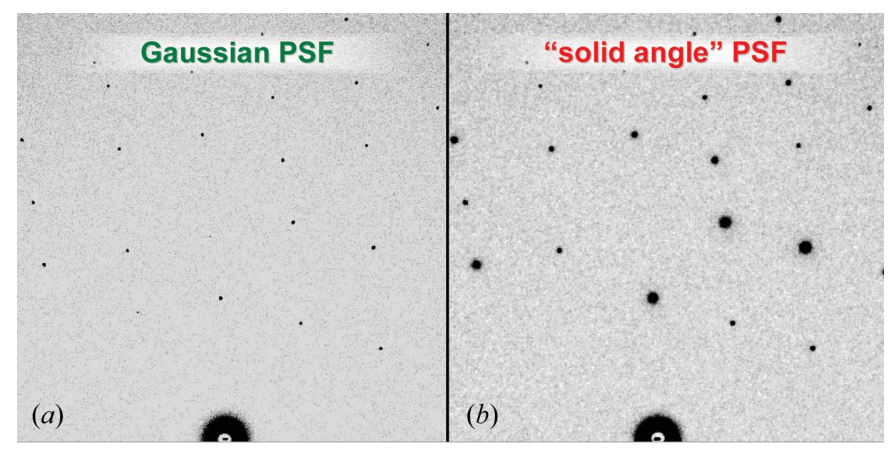

Figure 4

Simulated diffraction patterns from MLFSOM (Holton, 2008) with spots of various intensities blurred by either $(a)$ a Gaussian filter or $(b)$ the 'solid angle' PSF model proposed here. Both of these blurring filters have the same FWHM and the 16-bit images were histogram-equalized before display in $A D X V$ (Arvai, 2012; Szebenyi et al., 1997). The faint tails of the PSF make bright spots appear much larger than weak spots in $(b)$, but not in $(a)$.

material. However, since such small-angle contributions will also propagate through a protein crystal much like the rest of the incident beam, the overall PSF measured here is nevertheless directly relevant to protein crystallographic measurements, which are generally performed with some kind of beam-defining aperture. Indeed, applying this PSF to simulated diffraction patterns (Fig. 4) captures much of the visual appearance of spots on real images. Most of all, our observation that the PSF into 'severed' fibers is the same as that in 'intact' fibers excludes the possibility of significant contributions from pinhole fluorescence or SAXS.

No beam hardening is suspected with this set-up because the $\mathrm{Si}(333)$ reflection at $33423 \mathrm{eV}$ is well above the highenergy cut-off of the X-ray mirrors at $\sim 17 \mathrm{keV}$ (MacDowell et al., 2004), and we have previously verified the spectral purity of the beam with an identical optical set-up (Owen et al., 2009) at ALS beamline 12.3.1 [instrument described by Trame et al. (2004)] using an energy-resolving X-ray detector from Evex (Princeton, NJ, USA).

\subsection{Possible origins of the inverse-cube-law PSF}

The isolation of this inverse-cube dependence to the fiberoptic taper itself and the resemblance of the observed PSF to the solid angle subtended by a source $27 \mu \mathrm{m}$ above the pixel plane suggests a scattering mechanism. This is not unexpected. Since the fibers are in physical contact with the phosphor their numerical apertures are overfilled and every possible incidence angle up to the limit of total internal reflection is represented in the captured light. The limiting rays will continue to bounce back and forth down the fiber until they encounter a bend, which will increase some of the angles with the walls beyond the total-internal-reflection angle and so some of the light escapes. Scattering is therefore an unavoidable phenomenon in fiber-optic tapers, and indeed any optical system. The almost ubiquitous application of the Moffat function in astronomy and other fields is testament to this.

The origin of the inverse-cube-law dependence may then be explained by analogy with a point light source hovering $27 \mu \mathrm{m}$ 
over a plane of pixels. This system does produce an intensity distribution on the pixels given by $P$ from equation (2), and continues to do so even if the space between the light source and the pixels is filled with glass beads, or other strongly scattering but low-absorbing material. $P$ arises from conservation of energy, so as long as the medium is not strongly absorbing an inverse cube dependence is expected. Of course, the optical-fiber tapers are much longer than $27 \mu \mathrm{m}$, but the mean free path of a photon travelling through a fiber-optic taper is much longer in the direction of the fibers than it is in the directions crossing the fibers. Provided the number of 'scattering events' expected for an average photon moving down the taper axis from one end to the other is equal to that expected for a photon moving $27 \mu \mathrm{m}$ normal to the fiber axes (2.7 fiber diameters), then the taper can be regarded as a 'stretched' pile of glass beads, and $P$ still applies.

These tapers do include extra-mural absorbing fibers (EMA) to combat the effects of scattering, but cannot eliminate it entirely. It is possible that the EMA effect manifests as the slight 'wiggle' at about $500 \mu \mathrm{m}$ in the blue points on Fig. 2, but we have no way to confirm this. The wiggle could also be due a slight systematic offset in the 'zero' reference during our averaging process, but no attempt was made here to 'straighten' the data by applying an arbitrary offset.

\section{Conclusions}

The PSF of the popular fiber-coupled CCD X-ray detector design is neither Gaussian nor Lorentzian, but instead is better described as a Moffat function. The observation of identical PSF into 'severed' as well as 'intact' fibers strongly suggests that the 'tails' of the PSF arise from the scattering of visible light in the taper only, with little influence from the phosphor and the distribution of X-rays in the spot. Since the tails are not independent X-ray events, the visual appearance of 'bigger' spots as they grow brighter need not be detrimental to data quality. Specifically, we expect that by fitting an expression for the spot-PSF convolution as described here directly to pixel values will result in more accurate spot intensity integrals than those currently being obtained using conventional profile-fitting methods (which assume that the intensity of a pixel is due exclusively to X-ray photons falling directly upon it). A 'fitting approach' would eliminate systematic errors in background estimation arising from the tails and also suppress the influence of shot noise from X-ray photons falling on pixels outside the 'true' spot area.

The Gaussian component of the PSF $\left(w_{\mathrm{PSF}}\right)$ is estimated here to be $\sim 73 \mu \mathrm{m}$ after deconvoluting a beam size of $20 \mu \mathrm{m}$ and accounting for finite pixel size as described above. This is far too short to effectively discern a difference between 'intact' and 'severed' fibers, so the relative contribution of the taper and phosphor to this aspect of the PSF cannot be separated here. However, previous reports (Gruner et al., 2002) revealed that changing phosphor thickness does have an effect on the PSF, as does more aggressive EMA strategies (such as cladding each fiber in black), so it is expected that both phosphor and fiber contribute to $w_{\text {PSF }}$.
Admittedly, the measurements presented here were taken from only a single detector, but, since all commercially available fiber-coupled CCD detectors use tapers made by the same company (Incom, Charlton MA, USA) using similar EMA, it is expected that at least the functional form of the PSF presented here is generally applicable.

We would like to thank Gerd Rosenbaum, Robert Fischetti, Roger Durst, Sol Gruner and Michael Blum for extremely helpful discussions of this manuscript. This work was supported by grants from the National Institutes of Health (GM073210, GM082250 and GM094625) and the Integrated Diffraction Analysis Technologies (IDAT) program under Contract Number DE-AC02-05CH11231 with the US Department of Energy at Lawrence Berkeley National Laboratory.

\section{References}

Arndt, U. W. \& Wonacott, A. J. (1977). The Rotation Method in Crystallography: Data Collection from Macromolecular Crystals. Amsterdam: North-Holland.

Arvai, A. (2012). ADXV - a program to display $X$-ray diffraction images, http://www.scripps.edu/ arvai/adxv.html.

Bourgeois, D., Moy, J. P., Svensson, S. O. \& Kvick, Å. (1994). J. Appl. Cryst. 27, 868-877.

Cromer, D. T. \& Waber, J. T. (1965). Acta Cryst. 18, 104-109.

Gruner, S. M., Tate, M. W. \& Eikenberry, E. F. (2002). Rev. Sci. Instrum. 73, 2815-2842.

Hald, A. (1998). A History of Mathematical Statistics from 1750 to 1930. New York: Wiley-Interscience.

Hammersley, A. (1997). FIT2D. ESRF, Grenoble, France.

Holton, J. M. (2008). Acta Cryst. A64, C77.

Holton, J. M. \& Frankel, K. A. (2010). Acta Cryst. D66, 393-408.

Liaparinos, P. F., Kandarakis, I. S., Cavouras, D. A., Delis, H. B. \& Panayiotakis, G. S. (2006). Med. Phys. 33, 4502-4514.

MacDowell, A. A., Celestre, R. S., Howells, M., McKinney, W., Krupnick, J., Cambie, D., Domning, E. E., Duarte, R. M., Kelez, N., Plate, D. W., Cork, C. W., Earnest, T. N., Dickert, J., Meigs, G., Ralston, C., Holton, J. M., Alber, T., Berger, J. M., Agard, D. A. \& Padmore, H. A. (2004). J. Synchrotron Rad. 11, 447-455.

McMaster, W. H., Del Grande, N. K., Mallett, J. H. \& Hubbell, J. H. (1969-1970). Compilation of X-ray Cross Sections, LLNL Report UCRL-50174. Lawrence Livermore National Laboratory, CA, USA.

Madden, T. J., McGuigan, W., Molitsky, M. J., Naday, I., McArthur, A. \& Westbrook, E. M. (2006). IEEE Trans. Nucl. Sci. 2, 729-734.

Maslen, E. N., Fox, A. G. \& O'Keefe, M. A. (1999). International Tables for Crystallography, Vol. C, Mathematical, Physical and Chemical Tables, 2nd ed., Table 6.1.1.4, Coefficients for analytical approximation to scattering factors. Dordrecht: Kluwer.

Moffat, A. F. J. (1969). Astron. Astrophys. 3, 455-461.

Moivre, A. de (1738). The Doctrine of Chances: or A Method of Calculating the Probabilities of Evens in Play, 2nd ed. London: Woodfall.

Owen, R. L., Holton, J. M., Schulze-Briese, C. \& Garman, E. F. (2009). J. Synchrotron Rad. 16, 143-151.

Schreurs, A. M. M., Xian, X. \& Kroon-Batenburg, L. M. J. (2010). J. Appl. Cryst. 43, 70-82.

Szebenyi, D. M. E., Arvai, A., Ealick, S., LaIuppa, J. M. \& Nielsen, C. (1997). J. Synchrotron Rad. 4, 128-135.

Trame, C., MacDowell, A. A., Celestre, R. S., Padmore, H. A., Cambie, D., Domning, E. E., Duarte, R. M., Kelez, N., Plate, D. W., Holton, J. M., Frankel, K., Tsutakawa, S., Tsuruta, H., Tainer, J. A. \& Cooper, P. K. (2004). AIP Conf. Proc. 705, 502-505.

Vand, V., Eiland, P. F. \& Pepinsky, R. (1957). Acta Cryst. 10, 303-306. 